\title{
La comunicación interna y el desarrollo humano en el sector restaurantero regional en Yucatán, México
}

Internal communication and human development in the regional restaurant sector in Yucatán, Mexico

A comunicação interna e o desenvolvimento humano no setor de restaurantes regional em Yucatán, México

Rebeca Illiana Arévalo Martínez

- Doctora en Comunicación Aplicada por la Universidad Anáhuac México.

- Profesora investigadora del Centro de Investigación para la Comunicación Aplicada (CICA) de la Facultad de Comunicación de la Universidad Anáhuac México

- Miembro del Sistema Nacional de Investigadores (SNI) Nivel 1 de CONACYT

- E-mail: rebeca.arevalo@anahuac.mx

Carlos Gaspar Pérez Várguez

- Maestro en Ciencias de la Educación por la Universidad Anáhuac Mayab.

- $\quad$ Profesor de la Universidad Anáhuac Mayab

- Doctorando del Doctorado en Investigación de la Comunicación de la Universidad Anáhuac México

- E-mail: carlos.perez@anahuac.mx 


\section{Resumen}

La gastronomía yucateca es uno de los bienes culturales distintivos de México. Por ello, se realizó un estudio cuantitativo con tres restaurantes de comida regional de Mérida, Yucatán, en los cuales se entrevistó a sus colaboradores para determinar si la comunicación está relacionada con la conformación de una cultura organizacional que genere representaciones sociales vinculadas a las tradiciones regionales y al crecimiento de los colaboradores en términos de bienestar para el desarrollo humano. Se eligió este giro ya que en Yucatán el sector restaurantero regional tiene un lugar preponderante en la economía local.

PALABRAS CLAVE: COMUNICACIÓN INTERNA • CULTURA ORGANIZACIONAL・REPRESENTACIONES SOCIALES •IDENTIDAD ORGANIZACIONAL - BIEN CULTURAL.

\section{Abstract}

Yucatan's gastronomy is one of the distinctive cultural assets of Mexico. Because of this, a quantitative study was conducted with three regional food restaurants in Mérida, Yucatán, in which their collaborators were interviewed to determine if the communication is related to the conformation of an organizational culture that generates social representations linked to the regional traditions and the growth of employees in terms of welfare for human development. This turn was chosen since in Yucatan the regional restaurant sector has a preponderant place in the local economy.

KEYWORDS: INTERNAL COMMUNICATION • ORGANIZATIONAL CULTURE • SOCIAL REPRESENTATIONS • ORGANIZATIONAL IDENTITY • CULTURAL HERITAGE.

\section{Resumo}

A gastronomia yucateca é um dos bens culturais distintivos do México. Por isso, realizou-se um estudo quantitativo com três restaurantes de comida regional de Mérida, Yucatán, nos quais seus colaboradores foram entrevistados para determinar se a comunicação está relacionada com a conformação de uma cultura organizacional que gera representações sociais vinculadas às tradições regionais e ao crescimento dos colaboradores em términos de bem-estar para o desenvolvimento humano. Elegeu-se este turno já que em Yucatán o setor de restaurantes regional tem um lugar preponderante na economia local.

PALAVRAS-CHAVE: COMUNICAÇÃO INTERNA • CULTURA ORGANIZACIONAL • REPRESENTAÇÕNES SOCIAIS •IDENTIDADE ORGANIZACIONAL・ BEM CULTURAL. 
$\mathrm{D}$ iferentes estudios han demostrado la relevancia de las pequeñas y medianas empresas (PyMES) para la economía de los países en América Latina y Europa. No obstante que ese tipo de instituciones mantienen una estructura organizacional muy particular, la cual favorece esquemas de comunicación sólidos, en ocasiones la simplicidad de su conformación ha permitido prácticas de comunicación perniciosa y, en algunos casos, nulas.

Esa situación resulta relevante para analizarse debido a que la comunicación en las organizaciones es un factor esencial para el desarrollo de la competitividad, favorece la mejora y facilita el flujo de información para asegurar la productividad. Otorgándole la importancia indicada, se convierte en un elemento estratégico para la gestión organizacional.

El sector restaurantero es parte, en su mayoría, del segmento de las PyMES y en México la importancia de eso se ha incrementado por su contribución a la economía nacional como factor detonante del crecimiento, tanto por su impacto en la creación de empleos como por su injerencia en acuerdos comerciales y la internacionalización. Ese tipo de empresas contribuye con un 50\% del empleo de México y participan del 29\% del producto interno bruto (PIB). Así mismo, de acuerdo a la Dirección de Planeación y Análisis Económicos de la Secretaría de Fomento Económico (Sefoe), con datos del Censo Económico 2014 del Instituto Nacional de Estadística y Geografía (INEGI) (Secretaría de Fomento Económico, 2015), para Yucatán las PyMES representan el $99 \%$ de la economía del estado.

Considerando la relevancia que tienen en la economía del país, es inverosímil que ese tipo de empresas generalmente no cuentan con estrategias de comunicación claramente definidas. Eso está relacionado con que destinan sus presupuestos a otros rubros prioritarios para su desarrollo constante como sueldos y salarios, pagos a proveedores, obligaciones tributarias, quedando pocos recursos para invertir en estrategias de comunicación.

Esa situación ha repercutido negativamente en el sector restaurantero, detonando un incremento en la rotación de su personal (Ávila, 2015) y desprotegiendo las representaciones sociales vinculadas a la identidad con las tradiciones regionales.

Este artículo identifica algunas características del manejo de la comunicación en el sector restaurantero en Yucatán, México, a partir de un estudio realizado con tres restaurantes de comida regional. Esa información es relevante debido a que favorece un acercamiento a la realidad en ese tipo de organizaciones y a la prioridad que los empresarios otorgan a elementos como la comunicación interna, para establecer la incidencia en el fortalecimiento de la identidad cultural de sus colaboradores y el desarrollo humano bajo la perspectiva de la teoría de las capacidades, propuesta por Amartya Sen.

\section{MARCO TEÓRICO}

\section{1) Las representaciones sociales desde Serge Moscovici}

Serge Moscovici acuña el término representación social como aquellos sistemas de valores, nociones y prácticas que proporcionan a los individuos los medios para orientarse en el contexto social y material, para dominarlo. Es una organización de imágenes y de lenguaje (Perera Pérez, 2003).

En términos organizacionales, las representaciones sociales juegan un papel preponderante, ya que los colaboradores crean una serie de significados que conforman su cultura e identidad organizacional a partir de las representaciones. Para el caso en estudio - los restaurantes de comida regional-, es fundamental considerar ese papel, ya que alrededor de la organización se dan todas las tradiciones que conforman el patrimonio de la gastronomía yucateca y brindan una mística especial a los empleados y los establecimientos. 
Por ello, las representaciones sociales cumplen funciones que deben ser tomadas en cuenta en el momento de aplicar un modelo de comunicación. Perera Pérez (2003) propone como funciones básicas las siguientes: función de conocimiento, aquella que permite comprender y explicar la realidad; función identitaria, las representaciones ayudan a definir la identidad y permitir la salvaguarda de los grupos; función de orientación, las representaciones permiten la guía de los comportamientos; función justificatoria, esas representaciones permiten a posteriori justificar el comportamiento de los integrantes de la organización.

Desde esa perspectiva, eso se relaciona de manera directa con el sentido de pertenencia cuando se entiende este como la vinculación afectiva que se logra con la organización o en ese caso con el patrimonio cultural. Moscovici (Perera Pérez, 2003) explica los procesos de estructuración de las representaciones y en un primer momento se da lo que el autor llama objetivación y refiere al proceso por el que los elementos abstractos (gastronomía yucateca) se transforman en imágenes para ser cosificado y convertirse en algo familiar para el empleado. Eso lleva implícito la apropiación de los conocimientos relativos al objeto de la representación y a la significación que toman para el individuo, así como el que los organice y estructure. En ese caso, corresponde a identificarlos con los valores e identidad de la organización y al encontrarlos consistentes los hace propios a su existencia funcionando a partir de ese momento como categorías sociales de un lenguaje común.

\section{2) Las capacidades y el bienestar desde Amartya Sen}

De manera tradicional, la función social de los restaurantes de comida regional en Yucatán, México, puede vincularse con el desarrollo económico, sin embargo, el sector restaurantero aporta elementos importantes también al desarrollo tecnológico, social, sustentable y humano. Partiendo de eso, este artículo pretende analizar el enfoque del desarrollo humano desde la teoría de las capacidades de Amartya Sen y cómo el concepto de bienestar impacta en la identidad cultural de los colaboradores de los restaurantes y sus prácticas de comunicación.

Por ello, los empresarios deben considerar, además de los bienes físicos para sus colaboradores, el desarrollo de otro tipo de bienes, como pueden ser la felicidad, la motivación, la autorrealización y especialmente los que fomenten la identidad cultural y el conocimiento de las tradiciones del patrimonio cultural. En ese sentido, la comunicación es una estrategia que facilita esa identificación y mediante la cual las organizaciones pueden favorecer la integración y apropiación de las tradiciones de la gastronomía yucateca.

La teoría de las capacidades de Amartya Sen se enfoca en valorar la calidad de vida, considerando como noción básica el bienestar orientado a la libertad de la persona para lograr una vida autorrealizada. Cejudo Córdoba (2007) afirma que, de acuerdo a Amartya Sen, la persona tiene capacidades por naturaleza que puede potencializar mediante bienes que llama habilitaciones y acciones que denomina funcionamientos, los cuales favorecen que llegue a estados de valor para gozar de libertad.

En ese sentido, para los restaurantes de comida regional, las estrategias de comunicación pueden formar parte de las habilitaciones que generen funcionamientos en sus colaboradores para detonar la capacidad de tener una identidad cultural, que favorezca el patrimonio de un bien cultural y, por lo tanto, la identidad con la organización, la misión, producto y filosofía que rodea a los restaurantes de comida regional.

En eso radica la importancia de la teoría de las capacidades, la cual resalta al desarrollo humano en el sentido del bienestar y el funcionamiento como la manera en la que una persona vive en libertad orientando sus acciones a partir de elecciones sociales, de derecho y de desarrollo. Esos preceptos propuestos por Amartya Sen han sido considerados en los índices de informes de desarrollo humano que organismos internacionales han considerado. 
Las teorías económicas tradicionalmente habían centrado el concepto de bienestar en los elementos físicos como el sueldo, sin embargo, la gran aportación de Amartya Sen es el dar preponderancia al concepto de bienestar centrado en el desarrollo humano y en los significados a los que se refieren los bienes culturales intangibles, producto de la herencia cultural, los cuales son adoptados y modificados en muchos casos y permiten que la cultura se mantenga viva. La gastronomía se encuentra en esa clasificación y los empleados de los restaurantes deberían compartir como parte de su identidad esa cultura.

\section{3) Las prácticas de comunicación en las organizaciones}

Una organización se define como un producto de los seres humanos que fue creada con el fin de "producir bienestar en la sociedad y para satisfacer necesidades de las personas y grupos que habitan en el mundo social" (Rebeil Corella, 2009, p. 96), es decir, se trata de un sistema social vivo y abierto (Arévalo Martínez, 2014; Rebeil Corella, 2014). En el ámbito de la comunicación aplicada a la organización, la denominada comunicación organizacional fue sinónimo por mucho tiempo de comunicación interna (Rebeil Corella; Ruiz Sandoval Reséndiz, 1998). Pero también fue utilizado el término de manera indistinta para referirse a: la comunicación interna, externa o mercadológica (Rebeil Corella, 2008), como también a la comunicación institucional, integrada, corporativa etc., que se organizaban para lograr su operación y subsistencia en el mercado, para posteriormente convertirse en una función que integró tanto el ámbito de la comunicación mercadológica como de la comunicación interna. En ese sentido, la comunicación integrada (como se ha denominado en Brasil) incluye de manera sinérgica a la comunicación institucional, relaciones públicas, comunicación interna y comunicación mercadológica (Kunsch, 2016). Para los fines de este artículo, se retoma la definición de comunicación integral para las organizaciones que incluye:

Conjunto de conceptualizaciones teóricas y prácticas profesionales que tiene que ver con la Comunicación Organizacional, entendida ésta como la integración de la: comunicación creativa, comunicación mercadológica, comunicación corporativa, comunicación interna y comunicación administrativa en el contexto de tres distintos tipos de organizaciones: privadas, públicas y sociales y que contempla el estudio de las estrategias y las herramientas de la comunicación, así como el impacto de la misma y los procesos de retroalimentación.(Rebeil Corella, Hidalgo Toledo; Moreno Moreno, 2011)

En ese sentido, las prácticas de comunicación se refieren a todas aquellas tácticas que hacen posible la comunicación integral, cuyos componentes, conforme a la definición ya planteada, son los siguientes: comunicación creativa, la cual utiliza habilidades y talentos de los estrategas de comunicación para solucionar problemas e identificar áreas de oportunidad; comunicación mercadológica, dirigida a públicos externos, permite posicionar un mensaje, producto o servicio en la mente del consumidor; comunicación corporativa, que hace referencia a los mensajes que se proyectan a los públicos intermedios y externos sobre la misión y visión de la compañía; comunicación interna, aquella que se establece entre el público interno y los intermedios para garantizar la calidad del entorno laboral y sus interacciones; comunicación administrativa, la cual organiza los actos administrativos y relaciones dentro de la organización (Rebeil Corella, Hidalgo Toledo; Moreno Moreno, 2011).

Aunque "las organizaciones deben generar mensajes clave para cada una de estas cinco dimensiones de la comunicación y gestionarlos desde una visión de comunicación integral" (Rebeil Corella; Arévalo Martínez, 2017, p. 34), para fines de este artículo, es la comunicación interna la que se abordará, debido a que se pretende establecer la relación de la comunicación interna como un factor estratégico para las PyMES.

Dentro de la comunicación interna, aunque el principal público podría generalizarse y denominarse como empleados, se vislumbra la importancia de transmitir los mensajes de manera personalizada, a través de múltiples canales y diversos lenguajes de manera simultánea (Herrera; Arévalo Martínez, 2014). Por lo anterior, la comunicación debe lograr diálogos 
como base de la formación de relaciones de mayor solidez con el objetivo de asegurar una participación centrada en el bien común (Arévalo Martínez; Rebeil Corella, 2017). Las prácticas de comunicación interna de las PyMES deben abonar a la conformación de esas relaciones y generar vínculos entre sus empleados, sin descuidar también de los mensajes encaminados al aseguramiento de la operación y el logro de resultados.

Esa perspectiva del sentido humano del trabajo y particularmente de la comunicación debe resaltarse. Autores posmodernos han cuestionado la capacidad de diálogo y de construcción de discursos coherentes por parte de los empleados. Desde esa noción, la identidad es una construcción social que resulta de los diferentes discursos a los cuales las personas pueden estar expuestas. Es así que los colaboradores de una organización de ese giro reciben un sinnúmero de mensajes que deberían ir conformando su identidad de las tradiciones y no fragmentándola.

Si la identidad es una construcción social, la identidad será relativamente estable en sociedades homogéneas y relativamente estables con pocos discursos dominantes. En sociedades contemporáneas, heterogéneas, globales y teleconectadas los discursos disponibles se expanden enormemente. También cambian rápidamente. El individuo es hablado por tantos discursos que la fragmentación es virtualmente inevitable. (Alvesson; Deetz , 1996)

Con esa visión, es necesario entender a la comunicación colaborativa como aquel espectro que favorece la construcción de bienestar y desarrollo para los colaboradores, propiciando sinergia, trabajo en equipo y sobre todo el crecimiento personal de las personas al interior de la organización

Conforme a la teoría, se considera que las herramientas de comunicación adecuadas para la comunicación interna podrían ser el manual del empleado, las reuniones informativas, la revista o periódico interno, los tableros de avisos, el buzón de sugerencias, las circulares (Barquero Cabrero; Castillo Esparcia, 2011), entre otras. Sin embargo, en el caso de las PyMES, los mecanismos de comunicación informal suelen ser más comunes, por lo que:

para establecer mecanismos de información y transformar los canales informales de comunicación (boca a boca, almuerzos, etc), de las PyMES en canales formales (mails, carteleras) los actores intervinientes de las mismas deben sentir la necesidad de experimentar caudales de información en muchos casos similares a empresas grandes. (lurcovich, 2012, p. 82)

En el caso particular que nos ocupa -las PyMES del sector restaurantero de Mérida, Yucatán-, las prácticas en las que nos centraremos son las de comunicación interna, que están encaminadas en ese caso, a lograr que los empleados vivan la cultura organizacional, tengan orgullo de pertenecer a la organización, se sientan motivados y, con todo ello, puedan brindar un servicio de calidad que asegure no sólo la satisfacción de los clientes, sino que exceda sus expectativas. Si a todo lo anterior le agregamos la importancia que tiene en los restaurantes de comida regional, el respeto y orgullo nacional o regional que en ellos se transmite, la necesidad de una comunicación interna orientada hacia las tradiciones y su capital cultural, se genera un nuevo enfoque necesario de ponderar y modelar hacia prácticas de comunicación más especializadas.

\section{ANÁLISIS EMPÍRICO}

Este artículo reporta los resultados de un trabajo empírico para establecer las características del manejo de la comunicación en los restaurantes de comida regional y su relación con el fortalecimiento de la identidad cultural gastronómica de sus colaboradores para coadyuvar al desarrollo humano en las organizaciones. El estudio contempla un enfoque cuantitativo y brinda un mapeo de la situación que los restaurantes de ese tipo tienen con respecto al tema tratado aquí, lo cual no ha sido sistematizado por ningún estudio previo. 
Esta investigación provee de datos cuantitativos, que forman parte de una investigación más amplia realizada como trabajo doctoral y que pueden detonar otro tipo de análisis posteriores sobre las tradiciones como bien cultural, la comunicación interna y el desarrollo humano en las organizaciones desde la perspectiva de las capacidades. Para eso, a continuación, se detallan los instrumentos, muestra y análisis de la información.

El estudio cuantitativo se realizó a partir de tres hipótesis establecidas que son estudiadas mediante una encuesta aplicada en tres restaurantes de comida regional de Mérida, Yucatán, a 40 colaboradores que representan el total de la población de los establecimientos. La encuesta constó de 43 reactivos que midieron siete variables: prácticas de comunicación; mensajes clave; identidad organizacional; clima organizacional; cultura organizacional; sentido de pertenencia; representaciones sociales. La investigación buscó comprobar las siguientes hipótesis:

- H En los restaurantes de comida regional de Mérida, Yucatán, las prácticas de comunicación interna fortalecen la identidad organizacional, la cultura organizacional, el crecimiento profesional y las representaciones sociales.

- $\mathrm{H}_{2}$ Una cultura organizacional fuerte favorece la identidad organizacional, el crecimiento profesional y el sentimiento de orgullo y prestigio de los colaboradores de los restaurantes de comida regional en Mérida, Yucatán.

- $\mathrm{H}_{3}$ Una cultura organizacional fuerte favorece las representaciones sociales de la gastronomía como bien cultural en los colaboradores de los restaurantes de comida regional en Mérida, Yucatán.

\section{RESULTADOS}

En la Tabla 1, se reportan las estadísticas descriptivas de las variables analizadas correspondientes a prácticas de comunicación, cultura organizacional, prestigio y orgullo, tradiciones, crecimiento profesional, identidad organizacional y representaciones sociales. Puede notarse que los promedios más altos $(M=3.97, D E=0.64$ y $M=3.93, D E=0.56)$ corresponden a que los colaboradores están altamente identificados con la organización y sienten orgullo y prestigio de trabajar allá. Por otra parte, las variables prácticas de comunicación y cultura organizacional de los restaurantes presentan resultados por encima de la media de la escala (3) con valores de $M=3.40, D E=0.72$ y $M=3.50, D E=0.56$, respectivamente. En cuanto a las representaciones sociales y las tradiciones, ambas se encuentran por debajo de la media con valores de $M=2.82$, $D E=0.31$ y $M=2.61, D E=0.50$.

Tabla 1: Resultados descriptivos de variables

\begin{tabular}{|l|c|c|c|}
\hline & N & Media & Desviación estándar \\
\hline Prácticas de comunicación & 40 & 3.40 & .72 \\
\hline Cultura organizacional & 39 & 3.50 & .56 \\
\hline Prestigio y orgullo & 40 & 3.97 & .64 \\
\hline Tradiciones & 40 & 2.61 & .50 \\
\hline Crecimiento profesional & 40 & 2.45 & .95 \\
\hline Representaciones sociales & 40 & 2.82 & .31 \\
\hline Identidad organizacional & 40 & 3.93 & .56 \\
\hline
\end{tabular}

Nota: $N=40$ Las variables se midieron con un rango teórico de variación de 1 (nunca) a 5 (siempre). 
Para la variable "Prácticas de comunicación" en la Tabla 2, se presentan los resultados sobre los ítems que miden la formalidad de su implementación en los restaurantes. Entre los principales hallazgos, se destaca que los restaurantes no tienen actividades formales de comunicación interna que sean percibidas por los colaboradores como planeadas y que exista una inversión para ellas a diferencia de las actividades de comunicación externa. Reciben de manera eficiente información para realizar su trabajo, que se realiza de manera frecuente pero básicamente a través de comunicación interpersonal.

Tabla 2: Resultados descriptivos de escalas

\begin{tabular}{|l|c|c|}
\hline & Media & Desviación estándar \\
\hline $\begin{array}{l}\text { En el restaurante se planea de manera } \\
\text { constante la comunicación }\end{array}$ & 2.95 & 1.06 \\
\hline $\begin{array}{l}\text { Con qué frecuencia se utilizan los medios de comunicación } \\
\text { que tiene la institución establecidos con ustedes }\end{array}$ & 3.70 & 1.22 \\
\hline $\begin{array}{l}\text { Considera que las formas de comunicación utilizadas } \\
\text { por el restaurante cumplen su objetivo y son eficaces }\end{array}$ & 3.85 & 1.52 \\
\hline $\begin{array}{l}\text { Considera que la empresa invierte dinero } \\
\text { adecuadamente en comunicación interna }\end{array}$ & 2.17 & 1.63 \\
\hline
\end{tabular}

Nota: $N=40$ Las variables se midieron con un rango teórico de variación de 1 (nunca) a 5 (siempre).

Con respecto a la relación entre las variables, se estimó la regresión lineal simple de las prácticas de comunicación sobre la cultura organizacional, la identidad organizacional, el crecimiento profesional y las representaciones sociales, probándose la hipótesis de que las prácticas de comunicación están relacionadas con la cultura organizacional pero no con la identidad organizacional, las representaciones sociales y el crecimiento profesional. Se encontró que la pendiente $\beta=0.260, t(37)=2.141$, $p<.05$ fue estadísticamente significativa y, por lo tanto, se establece la relación lineal entre las prácticas de comunicación y la cultura organizacional. En ese caso, el valor de $r^{2}$ fue de .11 , indicando que aproximadamente un $11 \%$ del fortalecimiento de la cultura organizacional es explicado por las prácticas de comunicación. Sin embargo, en el caso de las representaciones sociales, la identidad organizacional y el crecimiento profesional se encontró que no existe una relación estadísticamente significativa quedando la pendiente de cada una de la siguiente manera: $\beta=0.58, \mathrm{t}(38)=0.838, p=.40 ; \beta=.077, \mathrm{t}(38)=.607$, $p=55 ; \beta=0.653, \mathrm{t}(38)=.961, p=.34 ; \mathrm{y}$ el valor de $r^{2}$ de $.018, .010$ y .161 , respectivamente.

Por otra parte, con relación a la cultura organizacional, se encontró que existe una correlación estadísticamente significativa con la identidad organizacional $\beta=0.569, \mathrm{t}(37)=3.96, p<.05$ y con el prestigio y orgullo $\beta=0.521, \mathrm{t}(37)=3.106, p<.01$, más no con las representaciones $\beta=.031, \mathrm{t}(37)=0.344, p=.73 \mathrm{ni}$ con el crecimiento personal $\beta=0.229, \mathrm{t}(37)=.830, p=.41 \mathrm{y}$ el valor de $r^{2}$ de $.313, .207, .003$ y .018 , respectivamente.

Finalmente, se consideró para el análisis el lugar de origen de los colaboradores debido a que éste podría resultar un factor determinante para que la identidad organizacional determinara las representaciones sociales, encontrándose que no fue estadísticamente significativo el lugar de origen, es decir, ser de Mérida, del interior o de otro estado de la República Mexicana $\beta=-.178, \mathrm{t}(18)=-1.411, p=.17 ; \beta=0.243, \mathrm{t}(11)=1.731, p=.11 ; \beta=0.286, \mathrm{t}(5)=1.059, p=.33$, con un valor de $r^{2}$ de $.100, .214 \mathrm{y} .428$.

\section{DISCUSIÓN}

La comunicación interna de los restaurantes analizados, en general, está enfocada hacia la creación de prestigio y orgullo entre sus colaboradores, así como también en la consolidación de su identidad organizacional. Sin embargo, a diferencia de 
lo que se plantea en la teoría, se observó que las representaciones sociales están muy abajo de la media, lo que significa que, en la práctica, éstas no juegan un papel preponderante en la comunicación interna de los restaurantes. Es decir, el hecho de que los colaboradores compartan algunos significados, los cuales dan forma a la cultura y la identidad organizacional no surge como resultado de un enfoque intencional a partir de las prácticas de comunicación interna.

También se destaca el hallazgo de que en último lugar se ubique tanto el crecimiento profesional como las tradiciones, lo cual deja de manifiesto que hace falta aún que esas organizaciones visualicen la importancia que tendría el detonar la identidad cultural entre sus colaboradores a partir de las tradiciones y del desarrollo humano. Lo anterior puede contrastarse con lo establecido por Serge Moscovici (Perera Pérez, 2003), cuando afirma que las representaciones sociales fortalecen la cultura y la identidad en las organizaciones.

En el estudio, se observó que la primera hipótesis se rechaza, pues, aunque sí se encontró correlación entre las prácticas de comunicación y la cultura organizacional, éstas no fortalecen la identidad organizacional, el crecimiento profesional y las representaciones sociales. La segunda hipótesis tampoco se comprobó, ya que, aunque se demostró la relación existente entre cultura organizacional e identidad organizacional, no hubo relación con el crecimiento profesional y el sentimiento de orgullo y prestigio de los colaboradores de los restaurantes que formaron parte de la muestra en Mérida, Yucatán. Sobre la tercera hipótesis, ésta tampoco se demostró pues no hubo una correlación estadísticamente significativa entre la cultura organizacional y las representaciones sociales de la gastronomía entre los colaboradores de los restaurantes analizados.

Los hallazgos confirman lo establecido por Amartya Sen (Cejudo Córdoba, 2007) acerca de los funcionamientos de la teoría de las capacidades y su función para detonar la capacidad de tener una identidad cultural y una identificación con los bienes culturales, ya que el resultado poco significativo con respecto a la implementación de prácticas de comunicación se relaciona con la casi nula asociación con las representaciones sociales y las tradiciones de la gastronomía yucateca en la población de estudio.

De igual forma, se confirma que existe una relación entre la cultura y la identidad organizacional, encontrándose que, en los establecimientos estudiados, se asocia la cultura organizacional con una alta identidad, prestigio y orgullo que sienten por la organización, confirmándose lo establecido por Maricela Perera Pérez (2003) cuando afirma que las funciones de las representaciones sociales fortalecen la identidad de las personas.

Las evidencias encontradas en los restaurantes estudiados confirman la teoría de Amartya Sen (Cejudo Córdoba, 2007) con respecto a que el desarrollo humano no está relacionado únicamente con las compensaciones económicas y físicas, sino más bien con las vinculadas al bienestar y su relación en ese caso con los bienes culturales. De igual forma, el análisis empírico comprobó que no es una variable significativa el origen de los colaboradores para asegurar la identidad organizacional.

\section{CONCLUSIONES}

Esta investigación muestra la necesidad que existe de fortalecer la planeación estratégica de la comunicación interna en los restaurantes de comida regional de Mérida, Yucatán, de manera que éstos sean capaces de generar prácticas de comunicación que aprovechen el patrimonio de la gastronomía yucateca y que potencialicen el desarrollo humano de sus colaboradores de manera objetiva y trascendente.

La cultura organizacional sólida que tienen los restaurantes, ligada a su identidad organizacional, se verá favorecida si logran incorporar las representaciones sociales de la gastronomía como parte del crecimiento profesional de los colaboradores, si 
lo hacen parte de sus prácticas cotidianas y con ello apuntalan el crecimiento del nivel de prestigio y orgullo que manifiestan sus integrantes.

Para lograr eso, se requiere de un modelo de comunicación interna que contemple estrategias para el fortalecimiento de una cultura centrada en la identidad organizacional y específicamente en las tradiciones de la gastronomía yucateca, ya que en la medida que los colaboradores establezcan representaciones sociales vinculadas al bienestar en términos de crecimiento profesional y personal no sólo desarrollarán sus capacidades humanas sino que fortalecerán la identidad del restaurante.

\section{REFERENCIAS}

ALVESSON, Mats; DEETZ, Stanley A. Critical theory and postmodernism approaches to organizational studies. In: CLEGG, Stewart R.; HARDY, Cynthia; LAWRENCE, Thomas B.; NORD, Walter R. Handbook of organization studies. London: Sage, 1996. p. 255-283.

ARÉVALO MARTÍNEZ, Rebeca Illiana. La construcción de imagen y reputación de las organizaciones vía Twitter. Correspondencias y Análisis, Surquillo, n. 4, p. 109-120, 2014. Disponível em: <https://goo.gl/G3EUsh>. Acesso em: 12 mar. 2017.

ARÉVALO MARTíNEZ, Rebeca Illiana; REBEIL CORELLA, Maria Antonieta. Presencia, interacción y responsabilidad social organizacional en las redes sociales digitales de organizaciones privadas en Iberoamérica. In: (Coords.). Responsabilidad social en la comunicación digital organizacional. Ciudad de México: Tirant Lo Blanch, 2017. p.17-51.

ÁVILA, Cinthya. 'Pega' a restaurantes rotación de personal. El Diario.mx, Juárez, 17 feb. 2015. Disponível em: <https://goo.gl/ fzMD2S>. Acesso em: 25 nov. 2017.

BARQUERO CABRERO, José Daniel; CASTILLO ESPARCIA, Antonio. Marco teórico y práctico de las relaciones públicas. Barcelona: Fundación Universitaria ESERP, 2011.

CARACCIOLO, Nadia. Comunicación para PyMES: cómo y porqué invertir en una gestión de prensa. Ituzaingó: Ugerman Editor, 2010.

CEJUDO CÓRDOBA, Rafael. Capacidades y libertad: una aproximación a la teoría de Amartya Sen. Revista Internacional de Sociología, Córdoba, v.65, n.47, p.9-22, 2007. Disponível em: <https://goo.gl/JCSkoQ>. Acesso em: 4 mar. 2017.

DI MAIO, Andrea. /l concetto di comunicazione: saggio di lessicografia filosofica e teologica sul tema di 'communicare' in Tommaso D'Aquino. Roma: Pontificia Universitá Gregoriana, 1998.

ENRIQUE JIMÉNEZ, Ana Maria; MORALES SERRANO, Francisca. Estructura de la comunicación empresarial. Pensar la Publicidad, Madri, v. 2, n. 2, p. 63-80, 2008.

ESPARZA AGUILAR, José Luis; GARCÍA PÉREZ DE LEMA, Domingo; DURÉNDEZ GÓMEZ GUILLAMÓN, Antonio. La cultura empresarial en la gestión de las empresas familiares: una aproximación teórica. Investigación y Ciencia, Aguascalientes, v. 18, n. 47, p. 13-20, abr. 2010.

GALVIS ORTIZ, Carlos Alberto; BOTERO MONTOYA, Luis Horacio. El comunicador corporativo y su accionar en las PyMES de Medellín. Signo y Pensamiento, Bogotá, v. 29, n. 56, p. 356-375. Disponível em: <https://goo.gl/ZWtJfN>. Acesso em: 6 abr. 2015. 
GÓMEZ GIRALDO, Juan Carlos; LÓPEZ JIMÉNEZ, Daniel Fernando; VELÁSQUEZ OSSA, César Mauricio. La naturaleza de la comunicación: un aporte a su discusión conceptual. Palabra Clave, Bogotá, v. 9, n. 1, p. 143-167, jun. 2006. Disponível em: $<$ https://goo.gl/vN6gly>. Acesso em: 15 abr. 2017.

GÓMEZ VILLANUEVA, Jorge; LLONCH, Andreu; RIALP CRIADO, Josep. Orientación estratégica, innovación y resultados en PyMES de nueva creación: el rol del marketing. Cuadernos de Gestión, Vizcaya, v. 10, p. 85-110, 2010. Disponível em: <https:// goo.gl/VGFYSW>. Acesso em: 10 out. 2015.

HERRERA, Marco V.; ARÉVALO MARTÍNEZ, Rebeca Illiana. Key leadership challenges and factors in public relations and communications in Mexico. In: BERGER, Bruce K.; MENG, Juan (Eds.). Public relations leaders as sensemakers: a global study of leadership in public relations and communication management. New York: Routledge, 2014. p. 262-277.

IURCOVICH, Patricia. La pequeña y mediana empresa y la función de la comunicación. Centro de Estudios en Diseño y Comunicación, Buenos Aires, n. 40, p. 79-84, 2012.

KUNSCH, Margarida M. Krohling. Comunicação organizacional estratégica. Rio de Janeiro: Summus, 2016.

MARÍN DUEÑAS, Pedro Pablo; GARCÍA GARCÍA, Maria. El estudio de la comunicación interna en el contexto del grado de publicidad y RR PP de la universidad de Cádiz. Historia y Comunicación Social, Madrid, v.18, p. 97-108, out. 2013. Número especial. Disponível em: <https://goo.gl/2vEjKy>. Acesso em: 5 out. 2015.

OCHOA JIMÉNEZ, Sergio; JACOBO HERNÁNDEZ, Carlos Armando; LEYVA OSUNA, Beatriz Alicia; LÓPEZ FIGUEROA, José Carlos. Estrategia, desempeño e identidad organizacional de las PyMES manufactureras mexicanas. Revista Internacional Administración \& Finanzas, Hilo, v. 7, n. 7, p. 75-90, 2014. Disponível em: <https://goo.gl/1PULDM>. Acesso em: 13 nov. 2015.

PERERA PÉREZ, Maricela. A propósito de las representaciones sociales: apuntes teóricos, trayectoria y actualidad. Havana: Centro de Investigaciones Psicológicas y Sociológicas, 2003. Disponivel em: <https://goo.gl/yhKpK3>. Acesso em: 22 fev. 2017.

RAMÍREZ SANDOVAL, Jorge Iván; GOCHICOA GRAMER, Enrique Frederico. Imagen corporativa: ventaja competitiva para las organizaciones PYME. Ciencia Administrativa, Xalapa, v. 2010-1, n. 1, p. 1-8, 2010. Disponível em: <https://goo.gl/eDcX2R>. Acesso em: 13 nov. 2015.

REBEIL CORELLA, Maria Antonieta. Subsistemas organizacionais e comunicação. In: KUNSCH, Margarida M. Krohling (Org.). Campos acadêmicos e aplicados de múltiplas perspectivas. São Caetano do Sul: Difusão, 2009. p. 95-109.

Organizaciones de telecomunicaciones en México: autoimagen y heteroimagen. In: CONGRESO DE LA ASOCIACIÓN LATINOAMERICANA DE INVESTIGADORES DE LA COMUNICACIÓN, 12., 2014, Lima. Anais... Lima: PUCP, 2014. Disponível em: <https://goo.gl/le8QaW>. Acesso em: 9 nov. 2017.

REBEIL CORELLA, Maria Antonieta; ARÉVALO MARTÍNEZ, Rebeca Illiana. Las organizaciones y sus procesos de comunicación: una visión integral. In: REBEIL CORELLA, Maria Antonieta; GUILLÉN OJEDA, Griselda (Coords.). La comunicación para las organizaciones en México: evolución, teoría y práctica. Ciudad de México: Tirant Lo Blanch, 2017. p. 25-40.

REBEIL CORELLA, Maria Antonieta; HIDALGO TOLEDO, Jorge Alberto; MORENO MORENO, Mariana. Gestión de la comunicación integrada en las organizaciones: competencias básicas para la formación del gestor. In: LEÓN DUARTE, Gustavo A. Estudios 
de la comunicación: estrategias metodológicas y competencias profesionales en comunicación. Ciudad de México: Pearson, 2011. p. 51-82.

REBEIL CORELLA, María Antonieta; RUIZ SANDOVAL RESÉNDIZ, Celia. El poder de la comunicación en las organizaciones. México: Plaza y Valdés, 1998.

RINCÓN QUINTERO, Yanyn. Comunicación corporativa, relaciones públicas y logística en la dinámica organizacional. Encuentros, n. 1, p. 47-58, jun. 2014. Disponível em: <https://goo.gl/9AgAq9>. Acesso em: 5 out. 2015.

SARSOSA PROWESK, Kewy; GÓMEZ, Diana Marcela. Características de la cultura organizacional y comunicación interna en una comercializadora de lácteos de Cali. Pensamiento Psicológico, Cali, v. 9, n. 17, p. 57-67, 2011. Disponível em: <https://goo. gl/Q99uup>. Acesso em: 3 out. 2015.

SECRETARIA DE FOMENTO ECONÓMICO. Perfil económico. 2015. Disponível em: <https://goo.gl/CHkKVq>. Acesso em: 9 mar. 2018.

TERÁN VALERA, Omar Ernesto; LORENZO IRLANDA, José. Influencia de la cultura organizacional en el desempeño laboral y la productividad de los trabajadores administrativos en instituciones de educación superior. Omnia, Maracaibo, v. 17, n. 1, p. 96-110, jan./abr. 2011. Disponível em: <https://goo.gl/ZRzV95>. Acesso em: 27 out. 2015.

YEPES STORK, Ricardo. Qué es eso de la filosofía: de Platón a hoy. Barcelona: Del Drac, 1996.

Artículo recibido el 13.07.2017 e aprobado el 15.10.2017. 